\title{
Clinical Experience of Sirolimus Regarding Efficacy and Safety in Systemic Lupus Erythematosus
}

OPEN ACCESS

Edited by:

Cecilia Beatrice Chighizola, Istituto Auxologico Italiano (IRCCS),

Italy

Reviewed by:

Matteo Piga,

Università degli Studi di Cagliari, Italy

Micaela Fredi,

ASST degli Spedali Civili di Brescia,

Italy

*Correspondence:

Christopher Sjöwall

christopher.sjowall@liu.se

orcid.org/0000-0003-0900-2048

Specialty section:

This article was submitted to Inflammation Pharmacology,

a section of the journal

Frontiers in Pharmacology

Received: 15 November 2018

Accepted: 21 January 2019

Published: 06 February 2019

Citation:

Eriksson P, Wallin $P$ and Sjöwall C (2019) Clinical Experience of Sirolimus

Regarding Efficacy and Safety

in Systemic Lupus Erythematosus.

Front. Pharmacol. 10:82

doi: 10.3389/fphar.2019.00082

\section{Per Eriksson, Philip Wallin and Christopher Sjöwall*}

Rheumatology/Division of Neuro and Inflammation Sciences, Department of Clinical and Experimental Medicine, Linköping University, Linköping, Sweden

New treatment options constitute unmet needs for patients diagnosed with systemic lupus erythematosus (SLE). Inhibition of the mammalian target of rapamycin (mTOR) pathway by sirolimus, a drug approved and in clinical use to prevent transplant rejection, has shown promising effects in lupus animal models as well as in patients with both antiphospholipid syndrome and SLE. Sirolimus inhibits antigen-induced T cell proliferation and increases the number of circulating regulatory $T$ cells. Recently, sirolimus was tested in an open label phase 1/2 trial, including 43 patients with active SLE, resistant or intolerant to conventional medications. The results were encouraging showing a progressive improvement, including mucocutaneous and musculoskeletal manifestations. At our university unit, we have more than 16 years' experience of sirolimus as treatment for non-renal manifestations of SLE. Herein, we retrospectively evaluated data on tolerance, dosage, affected organ systems, disease activity measures, corticosteroid reduction, concomitant immunosuppressive therapies, and patient-reported outcome measures (PROMs) such as pain intensity, fatigue, well-being and quality-of-life (QoL) in 27 Caucasian patients with mildly active SLE. Musculoskeletal manifestation was the main reason for sirolimus treatment followed by skin involvement and leukocytopenia. Mean time on sirolimus was 47.1 (range 2-140) months. Decreasing global disease activity was observed, as measured by the clinical SLE disease activity index-2000, with a mean reduction of 2.5 points (range -10 to 0 ) and a corresponding mean reduction of the physician's global assessment (0-4) of 0.64 (range -2 to 0 ). The mean daily dose of corticosteroids (prednisolone) was reduced by $3.3 \mathrm{mg}(-12.5$ to 0$)$. Non-significant trends toward improvements of QoL and pain intensity were found. Serious side-effects were not seen during sirolimus treatment, but early withdrawal due to nausea $(n=4)$ and non-serious infections $(n=2)$ appeared. This observational study, including longtime real-life use of sirolimus in SLE, is the largest to date and it essentially confirms the results of the recent phase 1/2 trial. Our data indicate that sirolimus is efficient in patients with musculoskeletal SLE manifestations, particularly arthritis and tendinitis. Further randomized controlled trials evaluating the potential benefits of sirolimus in SLE are warranted, but should aim to enroll patients with shorter disease duration, less accrued damage, and more diverse ethnicities. 


\section{INTRODUCTION}

Novel therapies aiding patients with systemic lupus erythematosus (SLE) constitute an unmet need since the available drugs often are limited to efficacy in certain disease phenotypes, and may have significant side-effects (Lateef and Petri, 2012). In fact, only few of the medications used in clinical practice today are approved for SLE, and several new candidate drugs have recently failed to meet their primary end-points in randomized controlled trials (Doria et al., 2017; Geh and Gordon, 2018). Instead, current therapeutic strategies for SLE mainly rely on clinical experience of older therapies used in other rheumatic conditions, or originate from the area of transplantation.

The pathogenesis of SLE is multifactorial. Genetic susceptibility and environmental factors play important roles and are accompanied by the involvement of $\mathrm{T}$ and $\mathrm{B}$ cells, dendritic cells, macrophages and neutrophils (Bengtsson and Rönnblom, 2017). The profound $\mathrm{T}$ cell dysfunction found in SLE has partly been attributed to activation of the mammalian target of rapamycin (mTOR), representing an intracellular serine/threonine receptor which regulates cell growth, proliferation and survival. mTOR is formed by a protein complex which includes mTORC1 and mTORC2 (Perl, 2016). mTORC1 drives the expansion of $\mathrm{T}$ helper (Th) type 1 cells, Th17 $\mathrm{T}$ cells, and CD4-CD8- (doublenegative) $\mathrm{T}$ cells. mTORC2, as well as mTORC1, inhibit the development of $\mathrm{CD} 4+\mathrm{CD} 25+\mathrm{FoxP} 3+\mathrm{T}$ regulatory cells. In addition, the differentiation of macrophages and dendritic cells is influenced by mTOR (Thomson et al., 2009; Perl, 2016).

Mammalian target of rapamycin is thus implicated in the pathogenesis of SLE in several ways. Patients with SLE have a reduced number of regulatory $\mathrm{T}$ cells $\left(\mathrm{T}_{\text {regs }}\right)$ with impaired suppressive activity (Banica et al., 2017; Kato and Perl, 2018). Follicular helper $\mathrm{T}$ (Tfh) cells are critical for germinal center formation and B cell activation. Th cells are expanded in SLE, and mTOR1 may be of importance for Tfh differentiation, although results are conflicting (Oaks et al., 2016). Furthermore, the B cell stimulating factor BAFF promotes B cell activation via mTOR activation (Ke et al., 2014), and inhibition of mTOR in plasmacytoid dendritic cells limits production of type I interferons, which has obvious implications for SLE (Cao et al., 2008; Bengtsson and Rönnblom, 2017).

Rapamycin, under the generic designation sirolimus, is a drug approved and in clinical use to prevent transplant rejection. Rapamycin has been shown to prevent the development of nephritis in lupus-prone mice (Warner et al., 1994; Lui et al., 2008). Just recently, sirolimus was tested in an open label phase 1/2 trial, including 43 patients with active SLE, resistant or intolerant to conventional medications. The results were encouraging showing a progressive improvement in several disease phenotypes, including mucocutaneous and musculoskeletal manifestations (Lai et al., 2018). In addition, involvement of the mTOR pathway in vascular lesions associated with the antiphospholipid syndrome (APS) has also been suggested and may be of high relevance also in SLE (Canaud et al., 2014). In a recently published case series of 16 patients with active or quiescent lupus nephritis where 7 had a previous history of malignancy, Yap and co-authors described an auspicious response to sirolimus treatment (Yap et al., 2018). However, to our knowledge, larger compilations on longterm real-life experience of sirolimus in SLE have so far not been reported.

At our university unit, we have more than 16 years' experience of sirolimus as treatment for non-renal manifestations of SLE. Herein, we systematically evaluated our retrospective sirolimus data in relation to tolerance, dosage, affected organ systems, disease activity measures, corticosteroid reduction, concomitant immunosuppressive therapies, and patient-reported outcome measures (PROMs). Reduction of global SLE disease activity as defined by the physician's global assessment (PGA) and the clinical SLE Disease Activity Index 2000 (cSLEDAI-2K) score (Scott, 1993; Uribe et al., 2004) constituted the primary outcome of the present study.

\section{MATERIALS AND METHODS}

\section{Patients}

The University Hospital in Linköping constitutes a tertiary referral center serving two other regional public hospitals in the county council of Östergötland, Sweden. At the Rheumatology outpatient clinic, we have long experience of monitoring patients with SLE by a prospective, structured follow-up program "KLURING" (Swedish acronym for Clinical LUpus Register In Northeastern Gothia), including registration of disease phenotypes, ongoing medication, and comorbidities (Frodlund et al., 2013).

As part of the KLURING cohort, a total of 27 patients with SLE, classified according to the 1982 American College of Rheumatology (ACR) criteria (Tan et al., 1982; Ighe et al., 2015), received sirolimus in daily doses of 1-3 mg between June 2002 and August 2018, and were followed from initiation of treatment until withdrawal, death or end of study period. All patients had previously been intolerant, or were judged as inadequate responders, to at least two disease-modifying anti-rheumatic drugs (DMARDs). Patient characteristics at the initiation of sirolimus treatment are further detailed in Table $\mathbf{1}$.

\section{Assessments}

Systemic lupus erythematosus disease activity was assessed by the use of PGA (graded 0-4) (Scott, 1993) and the cSLEDAI$2 \mathrm{~K}$ score (which excludes items for low complement and positive anti-dsDNA antibodies) (Uribe et al., 2004). Acquired organ damage, required to have been persistent for at least 6 months, was recorded by the Systemic Lupus International Collaborating Clinics (SLICC)/ACR damage index (SDI) encompassing damage in 12 defined organ systems (Gladman et al., 1996). Continuous data on PROMs were collected. The PROMs included data on quality-of-life (QoL) captured by the EQ-5D score (Leidl, 2009), functional ability estimated by the health assessment questionnaire (HAQ) (Lomi et al., 1995), as well as pain intensity, fatigue and well-being, all measured using the visual analog scale (VAS; graded 0-100 mm) (Hallert et al., 2003). 


\section{Laboratory Measurements}

Safety was continuously monitored by blood cell counts, liver enzymes (including alanine aminotransferase and aspartate aminotransferase), plasma creatinine, and blood lipids (including total cholesterol and triglycerides). Inflammatory and serological disease activity were followed by the erythrocyte sedimentation

TABLE 1 | Characteristics of the included patients at the start of sirolimus treatment.

\begin{tabular}{|c|c|}
\hline Patient characteristics & Mean (range) or $\%$ \\
\hline & All $(n=27)$ \\
\hline \multicolumn{2}{|l|}{ Background variables } \\
\hline Females & 100 \\
\hline Age (years) & $44.3(20-65)$ \\
\hline Duration of SLE (years) & $9.8(2-34)$ \\
\hline Weight (kg) & $65.6(47-93)$ \\
\hline Length $(\mathrm{cm})$ & $165.7(147-176)$ \\
\hline Caucasian ethnicity & 100 \\
\hline cSLEDAI (score) & $4.5(1-12)$ \\
\hline PGA (score) & $1.3(0-2)$ \\
\hline SDI (score) & $1.0(0-6)$ \\
\hline Number of fulfilled ACR criteria & $5.5(4-8)$ \\
\hline \multicolumn{2}{|l|}{ Concomitant medication } \\
\hline Prednisolone, daily dose (mg) & $7.5(0-20)$ \\
\hline Hydroxychloroquine & 59.2 \\
\hline Methotrexate & 7.4 \\
\hline Mycophenolate mofetil & 11.1 \\
\hline Warfarin & 14.8 \\
\hline Acetylsalicylic acid & 29.6 \\
\hline Statins & 0 \\
\hline \multicolumn{2}{|c|}{ Clinical phenotypes (ACR-82 definitions) } \\
\hline (1) Malar rash & 48.1 \\
\hline (2) Discoid rash & 18.5 \\
\hline (3) Photosensitivity & 63.0 \\
\hline (4) Oral ulcers & 22.2 \\
\hline (5) Arthritis & 100 \\
\hline (6) Serositis & 48.1 \\
\hline (a) Pleuritis & 48.1 \\
\hline (b) Pericarditis & 3.7 \\
\hline (7) Renal disorder & 25.9 \\
\hline (8) Neurologic disorder & 3.7 \\
\hline (a) Seizures & 3.7 \\
\hline (b) Psychosis & 0 \\
\hline (9) Hematologic disorder & 66.7 \\
\hline (a) Hemolytic anemia & 3.7 \\
\hline (b) Leukocytopenia & 37.0 \\
\hline (c) Lymphocytopenia & 44.4 \\
\hline (d) Thrombocytopenia & 14.8 \\
\hline (10) Immunologic disorder & 51.9 \\
\hline (a) Anti-dsDNA antibody & 44.4 \\
\hline (b) Anti-Smith antibody & 7.4 \\
\hline (11) IF-ANA & 100 \\
\hline
\end{tabular}

ACR, American College of Rheumatology; IF-ANA, immunofluorescence microscopy antinuclear antibodies; cSLEDAI-2K, clinical Systemic lupus erythematosus disease activity index 2000; SDI, SLICC/ACR damage index. rate (ESR), and plasma analyses of C-reactive protein (CRP), creatine phosphokinase (CK), complement protein $3(\mathrm{C} 3)$, and $4(\mathrm{C} 4)$.

\section{Statistics}

The GraphPad software (version 4.0; GraphPad Software Inc., San Diego, CA, United States) and the Python Language Reference (version 3.7, available at http://www.python.org, Python Software Foundation, Wilmington, DE, United States) were used for preparing figures and for statistical evaluation. Since the number of observations was different between many visits, repeated paired $t$-tests were used to examine differences in laboratory variables overtime and Wilcoxon's test for paired samples was used to evaluate disease activity scores. Correlation analysis was performed using Pearson's correlation coefficient. Two-tailed $p<0.05$ was considered significant.

\section{RESULTS}

\section{Patients Treated With Sirolimus}

As demonstrated in Table 2, 27 unique female SLE patients at our unit were prescribed sirolimus between June 2002 and August 2018 (study period). The mean daily dose was $1.5 \mathrm{mg}$ (range 13). Before start of sirolimus, the mean number of failed DMARDs was 3.6 (range 2-6). The mean time on sirolimus was 47.1 (range $2-140)$ months. Six of 27 (22\%) withdraw the drug due to nausea $(n=4)$ and non-serious infections $(n=2)$ before the 3-month evaluation visit (early cessation indicated by asterisks in Table 2 ), which was the reason why these six cases were excluded from efficacy analyses. At the last follow-up in August 2018, seven patients were still on treatment with sirolimus, and one individual (who had reached remission after 70 months on sirolimus) was not considered in need of the drug anymore; this corresponds to a drug survival of $38 \%$ regarding cases that passed the 3-month evaluation visit.

\section{Organ Manifestations}

As shown in Table 2, musculoskeletal involvement was the target for sirolimus treatment (96\%), followed by cutaneous lupus (37\%), and leukocytopenia (7\%). Regarding specific musculoskeletal manifestations, arthritis (54\%) was the dominating reason for sirolimus, but tendinitis (15\%) and arthralgia (31\%) were also common. Seven of 27 (26\%) patients had a history of renal involvement, but none had signs of active lupus nephritis at the initiation of sirolimus. 5 (19\%) had concomitant APS. Sirolimus was frequently combined with corticosteroids, hydroxychloroquine (HQ) and/or other DMARDs as indicated in Table 2.

\section{Efficacy}

Inflammatory and serological disease activity was followed over time by measurement of ESR, CRP, C3, C4, and CK. As illustrated in Figure 1, levels of C4 increased slightly over time, whereas CRP and ESR were rather stable. CK did not change significantly (not shown). As shown in Figure 2A, a decreased 
TABLE 2 | Individual descriptions of the 27 female pations.

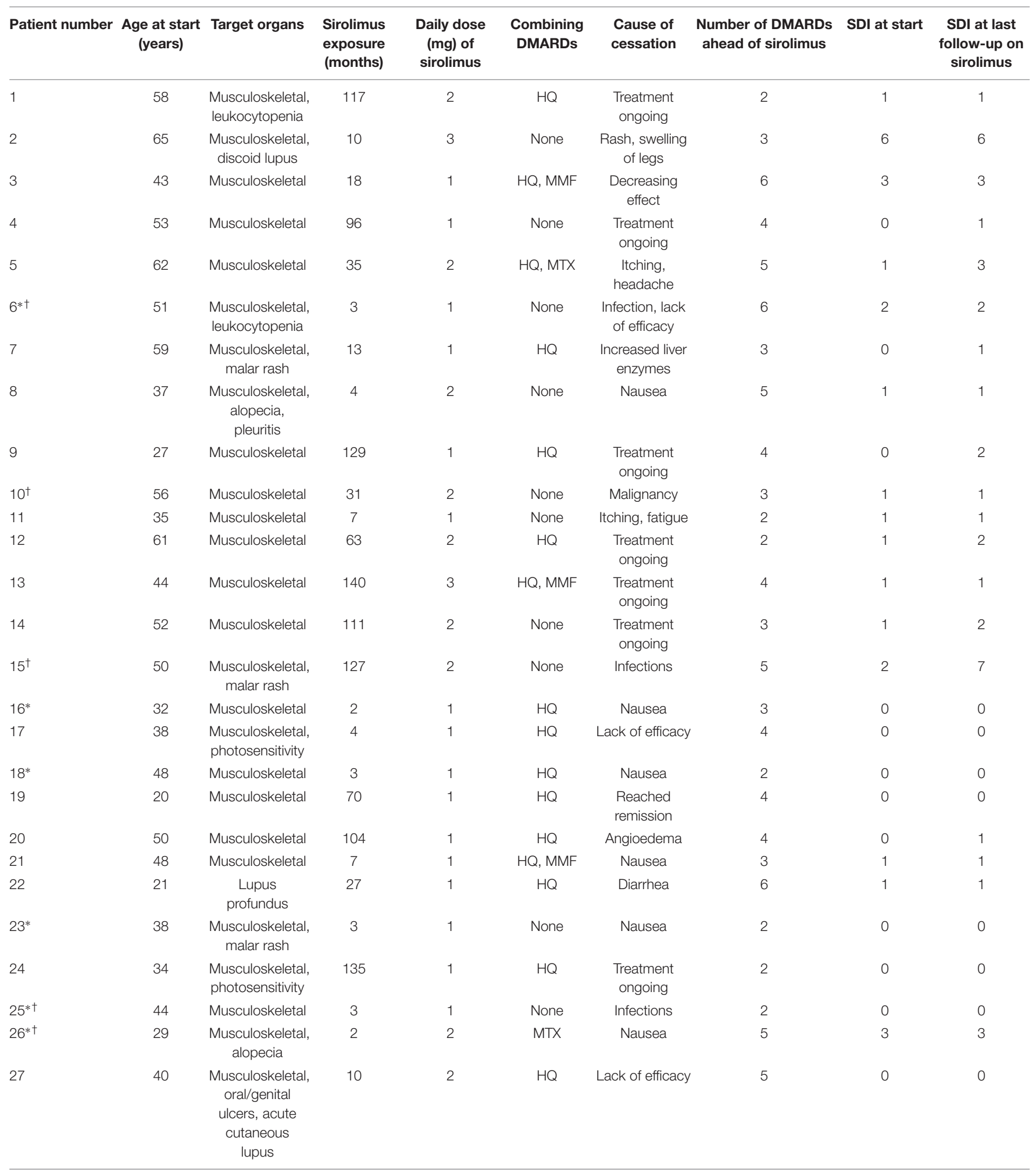

*Early cessation ( $\leq 3$ months). ${ }^{\dagger}$ Deceased at the end of study period. $H Q$, hydroxychloroquine; MMF, mycophenolate mofetil; MTX, methotrexate.

global disease activity was observed over time using cSLEDAI- A corresponding reduction of $0.64(-2$ to 0$)$ regarding PGA $2 \mathrm{~K}(p=0.0002)$ with a mean reduction of 2.5 (range -10 to 0$) \quad$ (Figure $2 B$ ) was also found $(p=0.0005)$. Sirolimus appeared comparing the time-point of initiation with the last observation. to be especially effective against arthritis and tendinitis, whereas 

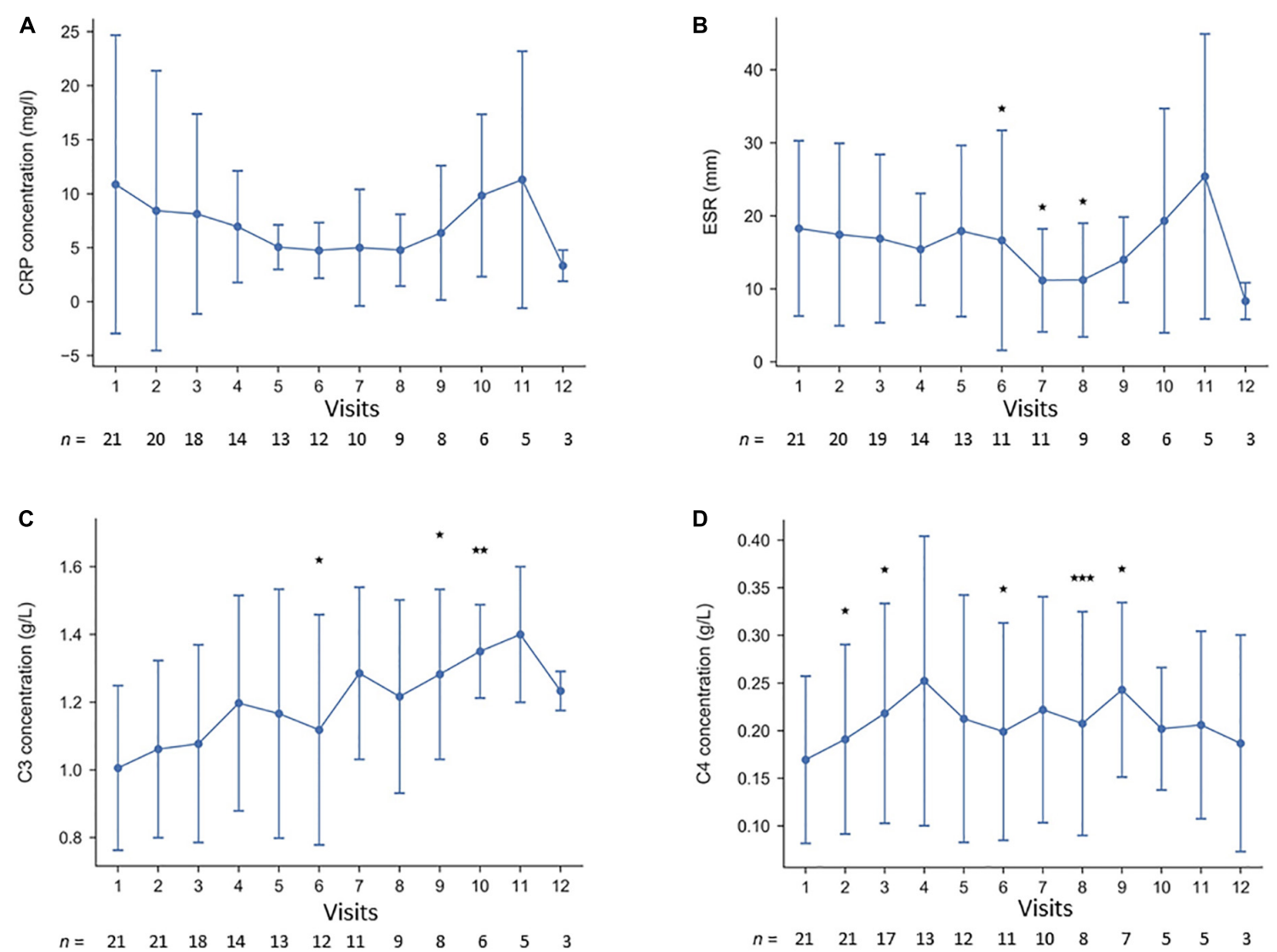

FIGURE 1 | (A-D) Longitudinal laboratory efficacy data at the first 12 visits of the 21 cases that passed the 3-month evaluation visit; (A) C-reactive protein, (B) erythrocyte sedimentation rate, (C) complement protein 3, and (D) complement protein $4 .{ }^{*} p<0.05,{ }^{* *} p<0.01$, and ${ }^{* * *} p<0.005$.

patients with arthralgia did not respond (Figure 2C). The mean daily dose of corticosteroids (prednisolone) at start was $7.5 \mathrm{mg}$ (Table 1), but it was reduced by $3.3 \mathrm{mg}$ (range -12.5 to 0 ) comparing the time-point of sirolimus initiation with the last observation $(p<0.001)$. The correlation between exposure to sirolimus and reduction of prednisolone dose was highly significant $(r=-0.7, p<0.0004)$ (Figure 2D). No significant improvements of PROMs (EQ-5D, HAQ, VAS pain intensity/fatigue/well-being) were observed. SDI scores at the initiation, and at the time-point of last follow-up on sirolimus, are demonstrated in Table 2. As shown in Table 1, the mean SDI at initiation of sirolimus was $1.0(0-6)$, and at last follow-up 1.5 (07 ). The mean annual accrual of SDI on sirolimus was 0.1 (range 0-0.9).

\section{Safety}

At end of the study period, 22 of 27 cases were alive. The five deceased patients (mean age 53.4 years, range 33-63) had been on sirolimus for a mean time of 33.2 months (range 2-133). The cause of death was malignancy in three cases (adenocarcinoma of the lung, ovarian cancer, acute myeloid leukemia) of which two patients had early cessations of sirolimus (before the 3month evaluation visit). Sepsis was the cause of death in the other two cases, whereof one patient had an early withdrawal. All five patients had discontinued sirolimus at the time-point of death, and none of the deaths were considered related to the drug. No renal flares, or onset of new lupus nephritis, were observed in any of the 27 patients.

No myocardial infarctions were registered, but two minor strokes were observed. Patient number 9, with SLE since 1983, was started on sirolimus because of arthritis in October 2007. Due to a new onset of seizure, a brain magnetic resonance imaging (MRI) was performed and showed new ischemic lesions. Antiphospholipid antibodies were detected and she was, in addition to SLE, diagnosed with APS which led to continuous treatment with warfarin. This event was indeed considered as an SLE exacerbation. However, the patient is still on sirolimus and has not had further strokes since then. Patient number 15, with multiple sclerosis since the 90s and SLE combined with APS since 2000, was started on sirolimus due to arthritis in June 2002. In 2009 , she developed a minor warfarin-dependent cerebrovascular bleeding but the treatment with sirolimus was not discontinued until 2013.

Drug safety was continuously monitored by blood tests. As demonstrated in Figure 3, no alarming signals regarding blood cell counts or renal function were noted. None of the patients developed hypercholesterolemia or triglyceridemia, leading to treatment with statins. After 13 months, patient number 7 ended 
A

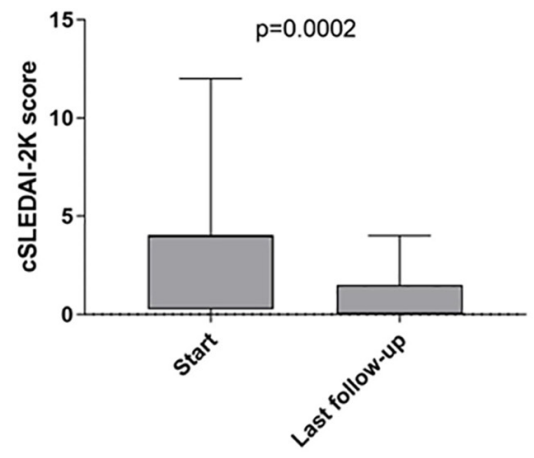

C

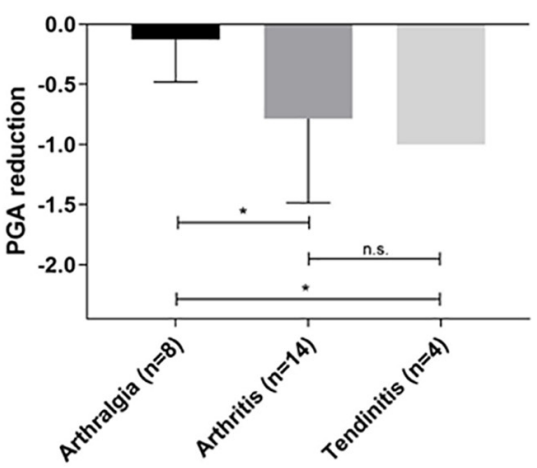

B

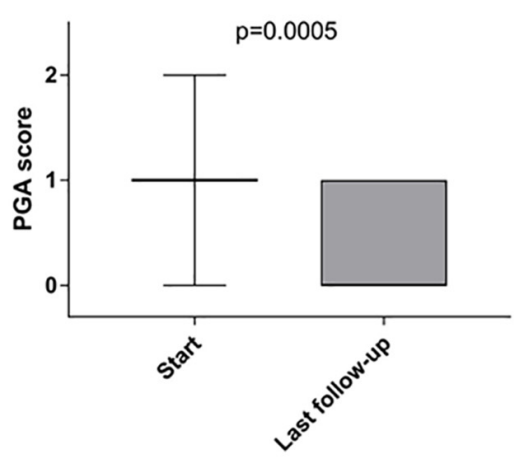

D

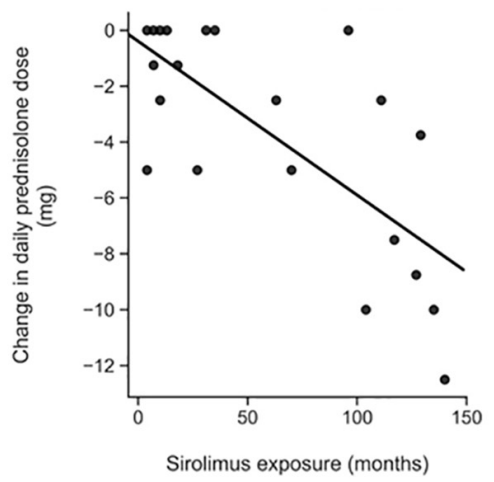

FIGURE 2 | (A-D) Differences in global disease activity between start/initiation of sirolimus therapy with regard to (A) clinical SLEDAl-2K, and (B) physician's global assessment (PGA). (C) Illustrates the reduction of PGA scores with regard to type of musculoskeletal manifestation. (D) Demonstrates the correlation between reduction of daily corticosteroid dose and the exposure of sirolimus in months. ${ }^{*} p<0.05$.

treatment with sirolimus due to elevated liver enzymes. However, both alanine aminotransferase and aspartate aminotransferase normalized shortly after cessation.

\section{DISCUSSION}

Current knowledge on the performance of sirolimus in autoimmune diseases is increasing, but observational data are mainly missing in rheumatology. Thus, we aimed to retrospectively compile the 16-years of clinical experience we have at our university unit on sirolimus in SLE. As a drug with potency of blocking $\mathrm{T}$ cell activation, sirolimus has clear-cut implications for the SLE pathogenesis. In support of sirolimus as a suitable treatment option in SLE, blockade of the mTOR pathway has shown promising effects in lupus animal models (Warner et al., 1994; Bonegio et al., 2005; Lui et al., 2008) as well as in patients (Fernandez et al., 2006; Yap et al., 2012, 2018; Lai et al., 2013; Bride et al., 2016; Herold et al., 2018). The recent phase $1 / 2$ trial showed effects primarily in the mucocutaneous and musculoskeletal organ systems (Lai et al., 2018). Reduced number of new episodes of rash was also reported but quite few patients developed cutaneous lupus over the study period why distinct conclusions were not possible. However, very interestingly, Lai et al. (2018) also observed that low levels of $\mathrm{T}_{\text {regs }}$ were reversed and high levels of interleukin (IL) 4 and IL-17 from other T cells than $\mathrm{T}_{\text {regs }}$ were reduced during the sirolimus treatment. Previously, Bride with coauthors reported beneficial effects of sirolimus on severe autoimmune cytopenias (Bride et al., 2016) and satisfactory response in individual patients with refractory discoid lupus erythematosus have been observed (Herold et al., 2018).

The Swedish healthcare system is tax funded and offers universal access, limiting the risks of patient selection bias, and drugs may be prescribed off label. As far as we know this observational study of sirolimus in SLE, including long time follow-up, is the largest to date. However, this is not a clinical trial and the included 27 patients with mildly active non-renal SLE were intolerant, or previously had an inadequate response, to at least two DMARDs. Some cases had tested multiple DMARDs without success and eventually failed on sirolimus as well. In addition, the study population was limited to cases without active renal or CNS involvement which is reflected by the rather low cSLEDAI-2K scores at start. Albeit, it is encouraging that none of our patients developed new (or incident) renal flares over the study period and the accrual of further damage was modest. Lack of longitudinal data on anti-dsDNA antibody levels and 28-joint disease activity scores constitute limitations of the study.

Comparing retrospective data with results from a clinical trial is challenging, but the outcome with reduced global disease activity (cSLEDAI-2K and PGA) essentially confirm the promising results of the phase $1 / 2$ trial (Lai et al., 2018) and 

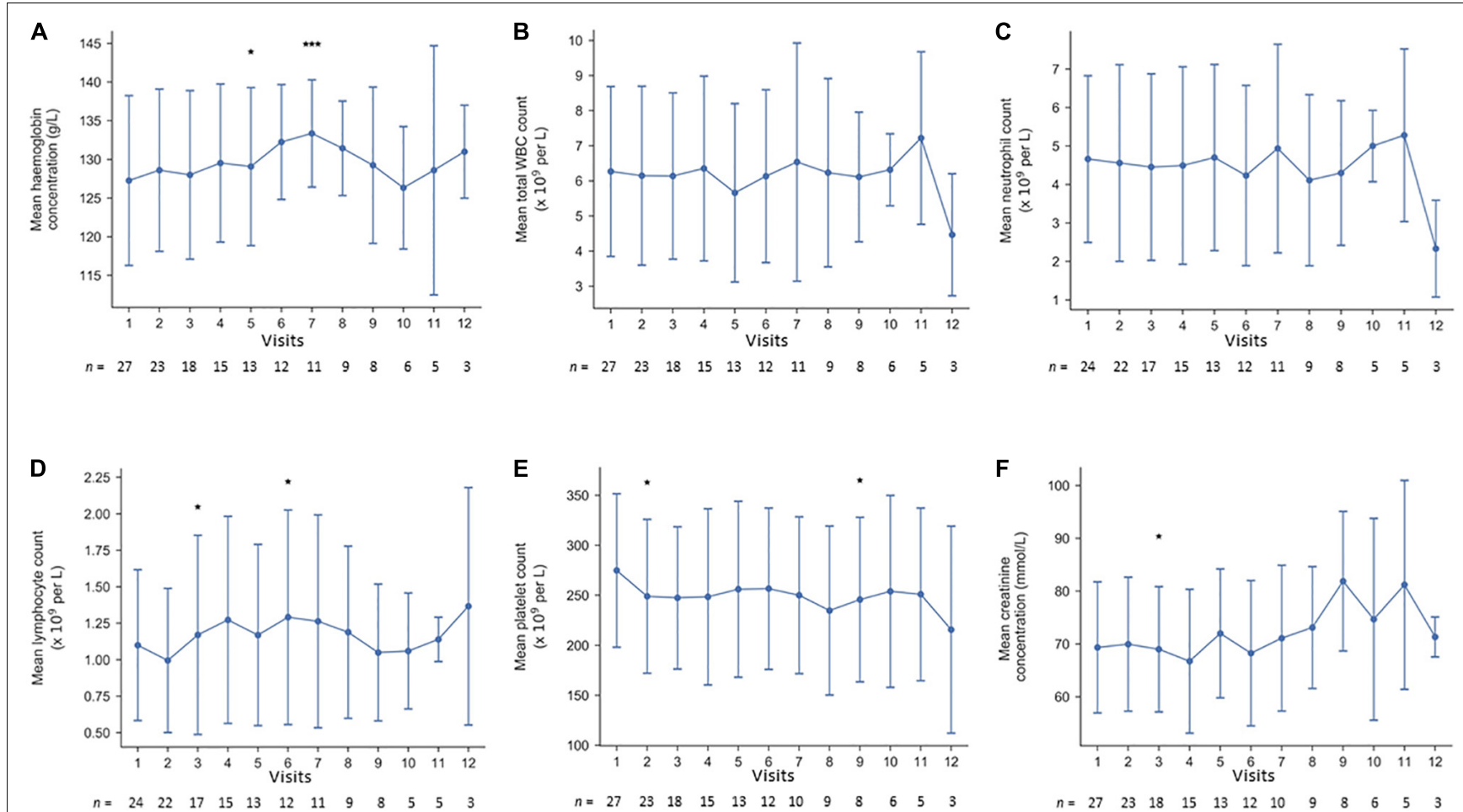

FIGURE 3 | (A-F) Longitudinal laboratory safety data at the first 12 visits of the 27 cases; (A) hemoglobin, (B) white blood cell count, (C) neutrophil count, (D) lymphocyte count, (E) platelet count, and (F) plasma creatinine. ${ }^{*} p<0.05,{ }^{* *} p<0.01,{ }^{* * *} p<0.005$.

indicate that sirolimus is efficient in patients with musculoskeletal manifestations (i.e., arthritis and tendinitis). Whereas $2 \mathrm{mg}$ sirolimus daily was used in the trial, we used slightly lower doses (mean $1.5 \mathrm{mg}$, with range $1-3 \mathrm{mg}$ ). Both dose regimens are lower than the doses usually prescribed in renal transplantation, a fact that has experimental support from lupus-prone mice (Warner et al., 1995).

The sirolimus trial did not include any PROMs (Lai et al., 2018). Although our patients with longtime follow-up showed trends toward improved QoL and less reported pain, the data were not statistically significant. The composition of the study population may be one of several reasons for this. Fifteen of $27(56 \%)$ patients were already affected by irreversible organ damage (SDI $>0$ ), which has a proven impact on both QoL and activity limitations in SLE (Björk et al., 2015). Failure of up to 6 DMARDs before the initiation of sirolimus probably also led to a bias in term of selection of refractory cases. Thus, for future studies, there may be better options to record improvements on QoL and other PROMs if cases with more recent-onset SLE were eligible.

Premature atherosclerosis in SLE may be related to type I interferons, whereas traditional risk factors seem to be of less importance (Kahlenberg and Kaplan, 2013; Leonard et al., 2018). Thus, pharmacological intervention preventing vascular events in SLE would obviously be of interest. Sirolimus inhibits smooth muscle hypertrophy in vessel walls (Gallo et al., 1999; Elloso et al., 2003), which may outweigh the transient hyperlipidemia sometimes reported in transplanted patients treated with higher doses of sirolimus (Asleh et al., 2018). mTOR signaling is also the major pathway in inhibition of endothelial autophagy which is implicated in atherogenesis (Xiong et al., 2014). Furthermore, as concomitant APS occurs in approximately one third of SLE patients and the mTOR pathway is involved in the vascular lesions related to APS, sirolimus may be of high importance regarding future studies of vascular disease in SLE (Canaud et al., 2014). Our retrospective case series do not permit any conclusions concerning vascular disease, but on the other hand neither hypercholesterolemia nor triglyceridemia were observed among our patients taking low doses of sirolimus. Another action of sirolimus with important implications for lupus nephritis and its longtime prognosis is the anti-fibrotic effects, possibly mediated via E-cadherein in experimental renal fibrosis (Liu, 2006).

A non-negligible proportion of the patients (>20\%) experienced non-serious side-effects or general discomfort and stopped sirolimus soon after its introduction. However, major side-effects were not seen and routine laboratory followup was normal in almost all cases. The rate of malignancies (11\%) may appear high, but none of them occurred during sirolimus therapy and in two of the cases exposure to the drug was very short. A causative effect of sirolimus is unlikely but cannot be entirely excluded. The question is also hampered by the fact that the longterm risk of several types of cancers in SLE is increased (Bernatsky et al., 2013). Data from organ transplant recipients show that longterm immunosuppressive regimens which include mTOR inhibitors are associated with an overall 
reduced cancer risk when compared to patients not treated with mTOR inhibitors (Yanik et al., 2015). In transplantation, however, use of sirolimus has been associated with pneumonitis, microangiopathy, thrombocytopenia, hypercholesterolemia, liver toxicity, lymphangioleiomyomatosis, and increased proteinuria in nephrotic patients (Marti and Frey, 2005; Takada et al., 2016). None of the above appeared in our series.

\section{CONCLUSION}

In summary, low doses of sirolimus were efficient in reducing global disease activity, especially regarding musculoskeletal manifestations, for patients with established mildly active SLE. Corticosteroids could be withdrawn or significantly reduced in many patients. Serious side-effects were not seen, although some patients stopped medication early due to non-serious discomfort. Only Caucasian patients were enrolled herein why it is warranted that further randomized controlled trials evaluating the potential benefits of sirolimus in SLE encompass larger and more mixed groups of cases.

\section{ETHICS STATEMENT}

In Sweden, drugs are allowed to be used off label. Nevertheless, informed consent was obtained from all subjects. The research

\section{REFERENCES}

Asleh, R., Briasoulis, A., Pereira, N. L., Edwards, B. S., Frantz, R. P., Daly, R. C., et al. (2018). Hypercholesterolemia after conversion to sirolimus as primary immunosuppression and cardiac allograft vasculopathy in heart transplant recipients. J. Heart Lung Transplant. 37, 1372-1380. doi: 10.1016/j.healun.2018. 07.004

Banica, L., Besliu, A., Pistol, G., Stavaru, C., Vlad, V., Predeteanu, D., et al. (2017). Dysregulation of anergy-related factors involved in regulatory $\mathrm{T}$ cells defects in systemic lupus erythematosus patients: rapamycin and vitamin D efficacy in restoring regulatory T cells. Int. J. Rheum. Dis. 19, 1294-1303. doi: 10.1111/ 1756-185X.12509

Bengtsson, A. A., and Rönnblom, L. (2017). Role of interferons in SLE. Best. Pract. Res. Clin. Rheumatol. 31, 415-428. doi: 10.1016/j.berh.2017.10.003

Bernatsky, S., Ramsey-Goldman, R., Labrecque, J., Joseph, L., Boivin, J. F., Petri, M., et al. (2013). Cancer risk in systemic lupus: an updated international multicentre cohort study. J. Autoimmun. 42, 130-135. doi: 10.1016/j.jaut.2012.12. 009

Björk, M., Dahlström, Ö, Wetterö, J., and Sjöwall, C. (2015). Quality of life and acquired organ damage are intimately related to activity limitations in patients with systemic lupus erythematosus. BMC Musculoskelet. Disord. 16:188. doi: 10.1186/s12891-015-0621-3

Bonegio, R. G., Fuhro, R., Wang, Z., Valeri, C. R., Andry, C., Salant, D. J., et al. (2005). Rapamycin ameliorates proteinuria-associated tubulointerstitial inflammation and fibrosis in experimental membranous nephropathy. J. Am. Soc. Nephrol. 16, 2063-2072. doi: 10.1681/ASN.2004030180

Bride, K., Vincent, T., Smith-Whitley, K., Lambert, M., Bleesing, J., Seif, A. E., et al. (2016). Sirolimus is effective in relapsed/refractory autoimmune cytopenias: results of a prospective multi-institutional trial. Blood 127, 17-28. doi: 10.1182/ blood-2015-07-657981

Canaud, G., Bienaime, F., Tabarin, F., Bataillon, G., Seilhean, D., Noël, L. H., et al. (2014). Inhibition of the mTORC pathway in the antiphospholipid syndrome. N. Engl. J. Med. 371, 303-312. doi: 10.1056/NEJMoa1312890 protocol was approved by the Regional Ethics Review Board in Linköping, Sweden (Decision No. M75-08/2008).

\section{AUTHOR CONTRIBUTIONS}

All authors were involved in drafting the article or revising it critically for important intellectual content and approved the final version to be published. CS had full access to all of the data in the study and takes responsibility for the integrity of the data and the accuracy of the data analysis. PE and CS conceived and designed the study. PE, PW, and CS acquired the data and analyzed and interpreted the data.

\section{FUNDING}

This work was supported by The Swedish Rheumatism Association, the King Gustaf V's 80-year Foundation, the King Gustaf V and Queen Victoria's Freemasons Foundation, and the County Council of Östergötland.

\section{ACKNOWLEDGMENTS}

The authors thank Senior Professor Thomas Skogh and Associate Professor Jonas Wetterö for comments on the manuscript.

Cao, W., Mannicassmy, S., Tang, H., Kasturi, S. P., Pirani, A., Murthy, N., et al. (2008). Toll-like receptor- mediated induction of type 1 interferon in plasmacytoid dendritic cells requires the rapamycin-sensitive PI(3)K-mTORp70S6K pathway. Nat. Immunol. 9, 1157-1164. doi: 10.1038/ni.1645

Doria, A., Cervera, R., Gatto, M., Chehab, G., and Schneider, M. (2017). The new targeted therapy in systemic lupus erythematosus: is the glass half-full or half-empty? Autoimmun. Rev. 16, 1119-1124. doi: 10.1016/j.autrev.2017.09.006

Elloso, M. M., Azrolan, N., Sehgal, S., Hsu, P. L., Phiel, K., Kopec, C. A., et al. (2003). Protective effect of the immunosuppressant sirolimus against aortic atherosclerosis in apo E-deficient mice. Am. J. Transplant. 3, 562-569. doi: 10.1034/j.1600-6143.2003.00094.x

Fernandez, D., Bonilla, E., Mizra, N., Niland, B., and Perl, A. (2006). Rapamycin reduces disease activity and normalizes $\mathrm{T}$-cell activation-induced calcium fluxing in patients with systemic lupus erythematosus. Arthritis Rheum. 54, 2983-2988. doi: 10.1002/art.22085

Frodlund, M., Dahlström, Ö, Kastbom, A., Skogh, T., and Sjöwall, C. (2013). Associations between antinuclear antibody staining patterns and clinical features of systemic lupus erythematosus: analysis of a regional Swedish register. BMJ Open 3:e003608. doi: 10.1136/bmjopen-2013-003608

Gallo, R., Padurean, A., Jayaraman, T., Marx, S., Roque, M., Adelman, S., et al. (1999). Inhibition of intimal thickening after balloon angioplasty in porcine coronary arteries by targeting regulators of the cell cycle. Circulation 99, 2164-2170. doi: 10.1161/01.CIR.99.16.2164

Geh, D., and Gordon, C. (2018). Epratuzumab for the treatment of systemic lupus erythematosus. Expert Rev. Clin. Immunol. 14, 245-258. doi: 10.1080/ 1744666X.2018.1450141

Gladman, D., Ginzler, E., Goldsmith, C., Fortin, P., Liang, M., Urowitz, M., et al. (1996). The development and initial validation of the systemic lupus international collaborating clinics/american college of rheumatology damage index for systemic lupus erythematosus. Arthritis Rheum. 39, 363-369. doi: 10.1002/art.1780390303

Hallert, E., Thyberg, I., Hass, U., Skargren, E., and Skogh, T. (2003). Comparison between women and men with recent onset rheumatoid arthritis of disease 
activity and functional ability over two years (the TIRA project). Ann. Rheum. Dis. 62, 667-670. doi: 10.1136/ard.62.7.667

Herold, M., Richmond, N. A., Montuno, M. A., Wesson, S. K., and Motaparthi, K. (2018). Rapamycin for refractory discoid lupus erythematosus. Dermatol. Ther. 31:e12631. doi: 10.1111/dth.12631

Ighe, A., Dahlström, Ö, Skogh, T., and Sjöwall, C. (2015). Application of the 2012 systemic lupus international collaborating clinics classification criteria to patients in a regional Swedish systemic lupus erythematosus register. Arthritis Res. Ther. 17:3. doi: 10.1186/s13075-015-0521-9

Kahlenberg, J. M., and Kaplan, M. J. (2013). Mechanisms of premature atherosclerosis in rheumatoid arthritis and lupus. Annu. Rev. Med. 64, 249-263. doi: 10.1146/annurev-med-060911-090007

Kato, H., and Perl, A. (2018). Blockade of Treg cell differentiation and function by the interleukin-21-mechanistic target of rapamycin axis via suppression of autophagy in patients with systemic lupus erythematosus. Arthritis Rheumatol. 70, 427-438. doi: 10.1002/art.40380

Ke, Z., Liang, D., Zeng, Q., Ren, Q., Ma, H., Gui, L., et al. (2014). hsBAFF promotes proliferation and survival in cultured B lymphocytes via calcium signaling activation of mTOR pathway. Cytokine 62, 310-321. doi: 10.1016/j.cyto.2013. 03.011

Lai, Z. W., Borsuk, R., Shadakshari, A., Yu, J., Dawood, M., Garcia, R., et al. (2013). Mechanistic target of rapamycin activation triggers IL-4 production and necrotic death of double-negative $\mathrm{T}$ cells in patients with systemic lupus erythematosus. J. Immunol. 191, 2236-2246. doi: 10.4049/jimmunol.1301005

Lai, Z. W., Kelly, R., Winans, T., Marchena, I., Shadakshari, A., Yu, J., et al. (2018). Sirolimus in patients with clinically active systemic lupus erythematosus resistant to, or intolerant of, conventional medications: a single-arm, openlabel, phase 1/2 trial. Lancet 391, 1186-1196. doi: 10.1016/S0140-6736(18) 30485-9

Lateef, A., and Petri, M. (2012). Unmet medical needs in systemic lupus erythematosus. Arthritis Res. Ther. 14(Suppl. 4):S4. doi: 10.1186/ar3919

Leidl, R. (2009). Preferences, quality of life and public health. Eur. J. Public Health 19, 228-229. doi: 10.1093/eurpub/ckp016

Leonard, D., Svenungsson, E., Dahlqvist, J., Alexsson, A., Ärlestig, L., Taylor, K. E., et al. (2018). Novel gene variants associated with cardiovascular disease in systemic lupus erythematosus and rheumatoid arthritis. Ann. Rheum. Dis. 77, 1063-1069. doi: 10.1136/annrheumdis-2017-212614

Liu, Y. (2006). Rapamycin and chronic kidney disease: beyond the inhibition of inflammation. Kidney Int. 69, 1925-1927. doi: 10.1038/sj.ki.5001543

Lomi, C., Burckhardt, C., Nordholm, L., Bjelle, A., and Ekdahl, C. (1995). Evaluation of a Swedish version of the arthritis self-efficacy scale in people with fibromyalgia. Scand. J. Rheumatol. 24, 282-287. doi: 10.3109/ 03009749509095164

Lui, S. L., Yung, S., Tsang, R., Chan, K. W., Tam, S., and Chan, T. M. (2008). Rapamycin prevents the development of nephritis in lupus-prone NZB/W F1 mice. Lupus 17, 305-313. doi: 10.1177/0961203307088289

Marti, H. P., and Frey, F. (2005). Nephrotoxicity of rapamycin - an emerging problem in clinical medicine. Nephrol. Dial. Transplant. 20, 13-15. doi: 10.1093/ ndt/gfh639

Oaks, Z., Winans, T., Huang, N., Banki, K., and Perl, A. (2016). Activation of the mechanistic target of rapamycin in SLE: explosion of evidence in the last five years. Curr. Rheumatol. Rep. 18, 73. doi: 10.1007/s11926-016-0622-8
Perl, A. (2016). Activation of mTOR (mechanistic target of rapamycin) in rheumatic disease. Nat. Rev. Rheumatol. 12, 169-182. doi: 10.1038/nrrheum. 2015.172

Scott, D. L. (1993). A simple index to assess disease activity in rheumatoid arthritis. J. Rheumatol. 20, 582-584.

Takada, T., Mikami, A., Kitamura, N., Seyama, K., Inoue, Y., Nagai, K., et al. (2016). Efficacy and safety of long-term sirolimus therapy for asian patients with lymphangioleiomyomatosis. Ann. Am. Thorac. Soc. 13, 1912-1922. doi: 10.1513/AnnalsATS.201605-335OC

Tan, E. M., Cohen, A. S., Fries, J. F., Masi, A. T., McShane, D. J., Rothfield, N. F., et al. (1982). The 1982 revised criteria for the classification of systemic lupus erythematosus. Arthritis Rheum. 25, 1271-1277. doi: 10.1002/art.1780251101

Thomson, A. W., Turnquist, H. R., and Raimondi, G. (2009). Immunoregulatory functions of mTOR inhibition. Nat. Rev. Immunol. 9, 324-337. doi: 10.1038/ nri2546

Uribe, A. G., Vilá, L. M., McGwin, G. Jr., Sanchez, M. L., Reveille, J. D., and Alarcón, G. S. (2004). The systemic lupus activity measure-revised, the mexican systemic lupus erythematosus disease activity index (SLEDAI), and a modified SLEDAI-2K are adequate instruments to measure disease activity in systemic lupus erythematosus. J. Rheumatol. 31, 1934-1940.

Warner, L. M., Adams, L. M., and Sehgal, S. N. (1994). Rapamycin prolongs survival and arrests pathophysiologic changes in murine systemic lupus erythematosus. Arthritis Rheum. 37, 289-297. doi: 10.1002/art.1780370219

Warner, L. M., Cummons, T., Nolan, L., and Sehgal, S. N. (1995). Sub-therapeutic doses of sirolimus and cyclosporine A in combination reduce SLE pathogenesis in the MRL mouse. Inflamm. Res. 44(Suppl. 2), S205-S206. doi: 10.1007/ BF01778335

Xiong, Y., Yepuri, G., Forbiteh, M., Yu, Y., Montani, J. P., Yang, Z., et al. (2014). ARG2 impairs endothelial autophagy through regulation of MTOR and PRKAA/AMPK signaling in advanced atherosclerosis. Autophagy 10, 2223-2238. doi: 10.4161/15548627.2014.981789

Yanik, E. L., Siddiqui, K., and Engels, E. A. (2015). Sirolimus effects on cancer incidence after kidney transplantation: a meta-analysis. Cancer Med. 4, 14481459. doi: 10.1002/cam4.487

Yap, D., Ma, M. K., Tang, C. S., and Chan, T. M. (2012). Proliferation signal inhibitors in the treatment of lupus nephritis: preliminary experience. Nephrology 17, 676-680. doi: 10.1111/j.1440-1797.2012.01646.x

Yap, D. Y. H., Tang, C., Chan, G. C. W., Kwan, L. P. Y., Ma, M. K. M., Mok, M. M. Y., et al. (2018). Longterm data on sirolimus treatment in patients with lupus nephritis. J. Rheumatol. 45, 1663-1670. doi: 10.3899/jrheum.180507

Conflict of Interest Statement: The authors declare that the research was conducted in the absence of any commercial or financial relationships that could be construed as a potential conflict of interest.

Copyright (c) 2019 Eriksson, Wallin and Sjöwall. This is an open-access article distributed under the terms of the Creative Commons Attribution License (CC BY). The use, distribution or reproduction in other forums is permitted, provided the original author(s) and the copyright owner(s) are credited and that the original publication in this journal is cited, in accordance with accepted academic practice. No use, distribution or reproduction is permitted which does not comply with these terms. 